Research Article

\title{
Fluid-Structure Interaction Analysis of Parachute Finite Mass Inflation
}

\author{
Xinglong Gao, Qingbin Zhang, and Qiangang Tang \\ School of Aerospace Science and Engineering, National University of Defense Technology, Changsha 410073, China \\ Correspondence should be addressed to Qiangang Tang; 441547742@qq.com
}

Received 18 January 2016; Accepted 6 April 2016

Academic Editor: Paul Williams

Copyright (C) 2016 Xinglong Gao et al. This is an open access article distributed under the Creative Commons Attribution License, which permits unrestricted use, distribution, and reproduction in any medium, provided the original work is properly cited.

Parachute inflation is coupled with sophisticated fluid-structure interaction (FSI) and flight mechanic behaviors in a finite mass situation. During opening, the canopy often experiences the largest deformation and loading. To predict the opening phase of a parachute, a computational FSI model for the inflation of a parachute, with slots on its canopy fabric, is developed using the arbitrary Lagrangian-Euler coupling penalty method. In a finite mass situation, the fluid around the parachute typically has an unsteady flow; therefore, a more complex opening phase and FSI dynamics of a parachute are investigated. Navier-Stokes (N-S) equations for uncompressible flow are solved using an explicit central difference method. The three-dimensional visualization of canopy deformation as well as the evolution of dropping velocity and overload is obtained and compared with the experimental results. This technique could be further applied in the airdrop test of a parachute for true prediction of the inflation characteristics.

\section{Introduction}

As a type of decelerator, parachutes have been widely used in recovery and life-saving systems. During the working of a parachute, its inflation is critical for the deceleration and has always been a challenge since the 1960s [1]. Fluid mechanics is an unsteady, viscous, and often compressible flow about a porous body with large shape changes. The parachute is represented as a tension structure that undergoes large transient deformations and is constructed using nonlinear materials with complex strain. All these disciplines are strongly coupled; therefore, converging the computations of fluid-structure interaction (FSI) coupling equations for inflating parachutes is difficult. Thus, some simplified dynamic inflation models have been developed to predict filling time and drag forces in parachutes $[2,3]$. Some are also integrated with ballistic flight-path equations to compute the trajectory and stability for the parachute-load system [4]. For the trajectory computation of a parachuteload system, the initial conditions are determined by the performance of parachute opening.

Generally, the process for parachute inflation can be sorted into infinite and finite mass conditions. When the unit area of a parachute bears a considerably heavy load, the deceleration during the opening process can be ignored and the inflow velocity kept constant. This is called infinite mass inflation. In contrast, the finite mass inflation implies significant deceleration during inflation. Benney and Stein investigated the time-variant aerodynamic characteristics of C-9 canopy by coupling the computational fluid dynamics (CFD) code to a mass-spring-damper structural dynamics (SD) code [5]. They then developed both FD and SD codes into a three-dimensional (3D) solver according to the deforming-spatial-domain/stabilized space-time procedure, which has been a popular FSI procedure for analyzing parachute inflation [6]. Further, the arbitrary LagrangianEuler (ALE) code is used as an efficient method to simulate the inflation process of a parachute. Tutt et al. developed FSI models for parachute inflation by using transient dynamic finite element code LS-DYNA [7]. Specifically, the finite mass simulation techniques were described; however, detailed FSI dynamic behaviors were not discussed further.

A front-tracking method based on a spring system offered an alternative to the modeling of the dynamic evolution of parachute canopy and load. The coupled algorithm that was named "impulse method" separates the impacts of the internal and external forces to eliminate nonphysical damping [8]. Moreover, an FSI method, combining a nonlinear 


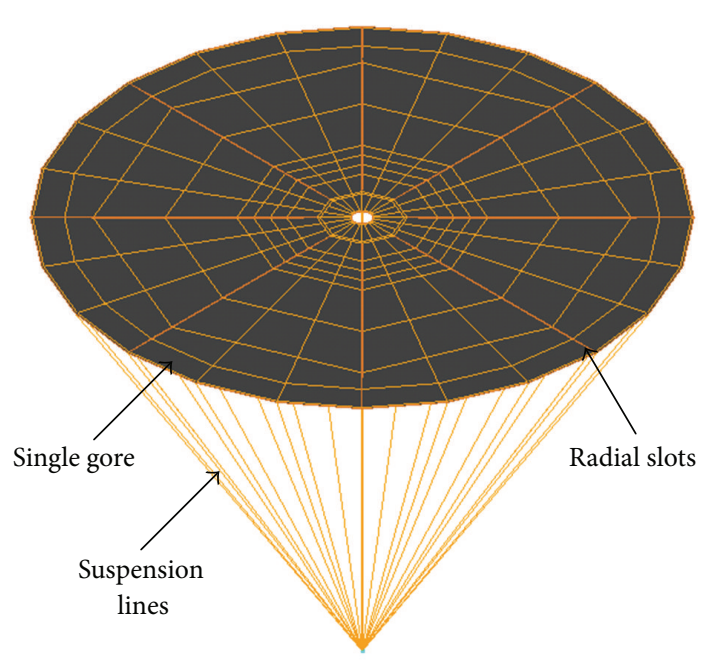

FigURE 1: Slot-parachute canopy.

finite element algorithm with a preconditioning finite volume method, and a simplified ALE FSI method were proposed to simulate the infinite mass inflation dynamics of a parachute. Although the FD equations were efficiently solved $[9,10]$, these studies do not contain any detailed information about the SD computation.

To predict the dynamic characteristics of a parachute during its descent and its inflation in an airdrop test, aerodynamics and flight mechanics have to be combined for each time step of simulation forces, and geometric replacements must be computed simultaneously [11]. However, because of the restrictions due to the complexity and time cost computation of parachute FSI equations, most simulation tools designed for parachute trajectory and landing process chose simplified aerodynamic models and coefficients as inputs [12]. Thus, we solved and obtained only some empirical equations or test data for the opening characteristics without fully considering the influence of the FSI phenomenon.

In this paper, we propose an efficient numerical approach for analyzing the inflation dynamics of a parachute-load system to be part of a high-fidelity simulation tool for an airdrop system. In addition, we investigated the inflation performance of a slot-parachute. The 3D shape deformation of the structure of the parachute during finite mass opening was predicted. Simultaneously, we solved the fluid mechanics around the canopy and reproduced them to analyze the evolvement mechanism of an unsteady flow during the deceleration of the parachute. Furthermore, we present the opening characteristics such as parachute drag, velocity, and pressure distribution in the terminal velocity state.

\section{Mathematical Model}

2.1. Simplifying Assumptions and Definitions. In this section, we discuss the development of a slot-parachute model. It is a life-saving parachute with 24 suspension lines and eight radial gaps symmetrically placed on the connecting area of eight gores (Figure 1). The parachute, which was stretched out from its pack, and the load were connected with a unit point mass. The load is represented by a dummy, which was used in the airdrop test. The following simplifying assumptions were considered:

(a) The packed geometry of a canopy before inflation is axially symmetric and no prestress exists.

(b) The opening process is a finite mass inflation considering the gravity.

(c) The air fluid is considered as an incompressible viscous flow at a low velocity.

(d) The fluid field is considered as a quasistate with a constant velocity at the inlet boundary.

\subsection{Governing Equation}

2.2.1. Structure Dynamics. The parachute components are mainly flexible and continuous media. Let $\boldsymbol{\Omega}^{s}$ be the spatial domain where superscript " $s$ " implies the structure, and let $\partial \boldsymbol{\Omega}_{s}$ denote the boundary of $\boldsymbol{\Omega}^{s}$. The governing equation of the structure is

$$
\rho_{s} \frac{d^{2} \mathbf{y}}{d t^{2}}=\nabla \cdot \sigma_{s}+\rho_{s} \mathbf{f}_{s}+\mathbf{g}
$$

where $\rho_{s}$ denotes the material density, $\mathbf{y}$ is the velocity vector of structure media, $\boldsymbol{\sigma}_{s}$ is the Cauchy stress tensor, $\mathbf{f}_{s}$ represents the external body forces acting on the structure, and $\mathbf{g}$ is the gravitational acceleration vector.

The canopy is made of a fabric with large deformation and nonlinear dynamic characteristics. A special stress-strain relation for formulating the composite membrane element is given by

$$
\begin{aligned}
\varepsilon_{1} & =\frac{1}{E_{1}}\left(\sigma_{1}-v_{1} \sigma_{2}\right), \\
\varepsilon_{2} & =\frac{1}{E_{2}}\left(\sigma_{2}-v_{2} \sigma_{1}\right), \\
2 \varepsilon_{12} & =\frac{1}{G_{12}} \tau_{12}+\alpha \tau_{12}^{3},
\end{aligned}
$$

where $\sigma, v$, and $E$ represent the longitudinal stress, Poisson's ratio, and elastic modulus (subscript 1 means longitudinal direction and 2 means traverse direction), respectively. $\tau_{12}$ is the shear stress, $G_{12}$ is the shear elasticity, and $\alpha$ is the nonlinear coefficient, which can be measured by the stressstrain relation test.

The ropes were mainly acted upon by the drag force contributed by the canopy. By considering the damping and nonlinear characteristic of ropes, the dynamic governing equation is

$$
F= \begin{cases}0 & \varepsilon \leq 0 \\ p(\varepsilon)+C \cdot \dot{\varepsilon} & \varepsilon>0\end{cases}
$$

where $p(\varepsilon)$ represents the nonlinear tensile function of ropes and $C$ is the damping coefficient. The strain $\varepsilon$ can be written as

$$
\varepsilon=\frac{\Delta l}{l_{0}-l_{\mathrm{off}}} .
$$

2.2.2. Fluid Dynamics. The fluid field during parachute inflation is a time-variant spatial domain. Let $\Omega^{f}$ be the spatial domain and let $\partial \boldsymbol{\Omega}_{f}$ denote the boundary of $\boldsymbol{\Omega}^{f}$; then the 
Navier-Stokes (N-S) equations for incompressible flows are [13]

$$
\begin{aligned}
\rho\left(\frac{\partial \mathbf{u}}{\partial t}+\mathbf{u} \cdot \nabla \mathbf{u}+\mathbf{f}\right)-\nabla \cdot \boldsymbol{\sigma} & =0 & & \text { on } \mathbf{\Omega}^{f}, \\
\nabla \cdot \mathbf{u} & =0 & & \text { on } \mathbf{\Omega}^{f},
\end{aligned}
$$

where $\rho_{s}, \mathbf{u}, \mathbf{f}$, and $\boldsymbol{\sigma}$ are the density, velocity vector, external body force, and stress tensor, respectively. By introducing the ALE formulation combined with the fluid and material mesh, which can move freely, the N-S governing equation can be rewritten as

$$
\begin{aligned}
\frac{\partial \rho}{\partial t}+\rho \cdot \operatorname{div}(\mathbf{v})+(\mathbf{v}-\mathbf{w}) \nabla \cdot \rho & =0 \\
\rho \frac{\partial \mathbf{v}}{\partial t}+\rho(\mathbf{v}-\mathbf{w}) \cdot \nabla \cdot \mathbf{v} & =\operatorname{div}(\boldsymbol{\sigma})+\mathbf{f}, \\
\rho \frac{\partial \mathbf{E}}{\partial t}+\rho(\mathbf{v}-\mathbf{w}) \nabla \cdot \mathbf{E} & =\boldsymbol{\sigma} \cdot \nabla \mathbf{v}+\mathbf{f} \cdot \mathbf{v},
\end{aligned}
$$

where $\mathbf{v}, \mathbf{w}$, and $\rho$ are the fluid particle velocity, material mesh velocity in reference coordinates, and fluid density, respectively, and $\mathbf{E}$ is the specific internal energy. Obviously, the ALE formulation contains both the Euler and Lagrange formulations, and Dirichlet and Neumann-type boundary conditions are prescribed on $\left(\Gamma_{t}\right)_{q}$ and $\left(\Gamma_{t}\right)_{h}$, respectively, where $\left(\Gamma_{t}\right)_{q}$ and $\left(\Gamma_{t}\right)_{h}$ are complementary subsets of boundary $\Gamma_{t}$. The initial condition on the velocity is divergence-free.

2.2.3. Penalty Coupling Scheme. The FSI simulation for parachute finite mass inflation generally involves the complement of structural and fluid mesh, and it is impossible to implement a complete match between these two items. By using the Eulerian-Lagrangian penalty coupling algorithm, the coupling force can be applied on opposite directions of the FSI interface [15]. By considering the porosity of canopy fabric, the pressure of porous media can be derived from the Ergun equation [16] of shell as follows:

$$
\frac{d \mathbf{P}}{d \mathbf{r}}=a(\mu, \varepsilon) \cdot \mathbf{v}_{\mathrm{rel}}+b(\rho, \varepsilon) \cdot \mathbf{v}_{\mathrm{rel}}^{2},
$$

where $\mathbf{P}$ is the pressure, $\mathbf{r}$ is the normal direction of shell, and $\varepsilon$ is the porosity of material; the coefficient $a(\mu, \varepsilon)$ is the reciprocal permeability of the porous shell or viscous coefficient, and $b(\rho, \varepsilon)$ represents the inertia coefficient.

By using the explicit dynamic integral method, the velocities and pressures can be effectively solved, satisfying the continuity constraint in (7).

2.3. Generation of Numerical Models for FSI. The load implemented at the joint of the parachute was represented by a dummy body, and Figure 2 represents the finite element method (FEM) model of the parachute system. For the finite mass inflation, the computation domain should be sufficiently large to accommodate the dropping distance of the parachute within the filling time and reduce the influence of boundary reflecting as much as possible. The geometry of fluid domain is cylindrical and meshed by hexagons (Figure 3). The canopy geometry is designed by CATIA software and output into the FEM tool for meshing.
TABLE 1: Statistical information of FSI models.

\begin{tabular}{lccc}
\hline Computational term & Fluid & Canopy & Suspension lines \\
\hline Nodes & 441000 & 9072 & 3244 \\
Elements & 460664 & 9488 & 3077 \\
Materials & Solid & Shell & Beam \\
Density $\left(\mathrm{kg} / \mathrm{m}^{3}\right)$ & 1.18 & 583 & 4850 \\
Poisson's ratio & 0.13 & 0.13 & 0.13 \\
\hline
\end{tabular}

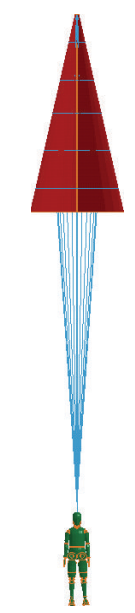

FIGURE 2: Construct of parachute-load system.

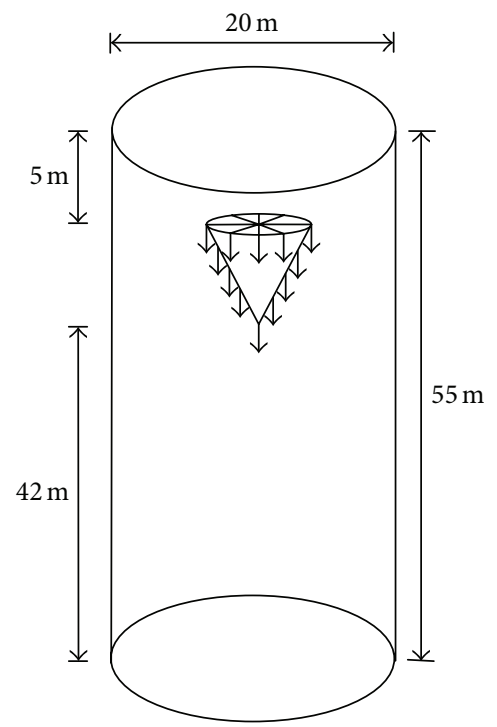

FIGURE 3: Geometrical shape of fluid domain.

The processing of elements primarily occupies the CPU time during the FSI computation. Because of the high cost of the ALE approach, the construction of FSI model was kept simple and frugal. The density of fluid mesh varied according to the placement of the parachute. Table 1 summarizes the $3 \mathrm{D}$ mesh information of the parachute, dummy, and fluid, and Table 2 presents the physical parameters of the dummy.

The level of refinement in a CFD mesh should be decided by the size of the structure. The 1:1 length of elements between the fluid and structure mesh is best for the accuracy and convergence of simulation. The grid density of the 


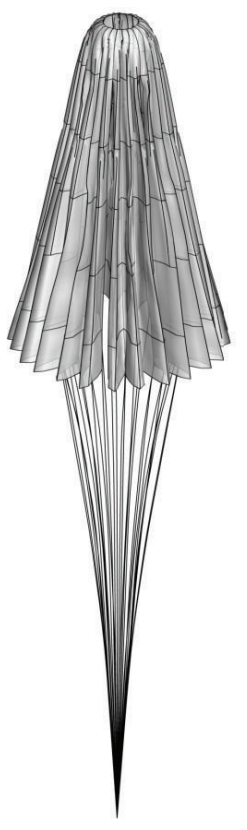

(a)

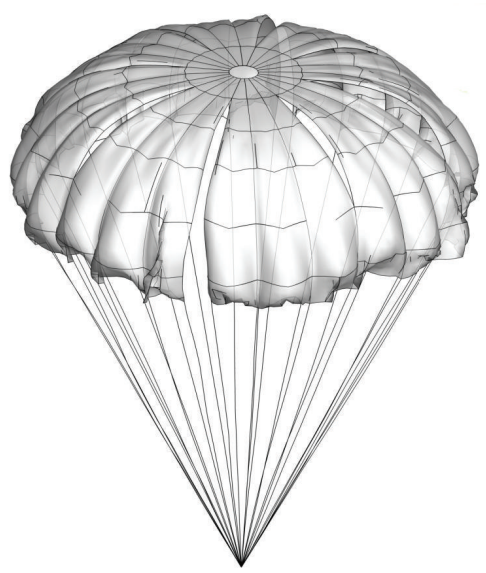

(d)

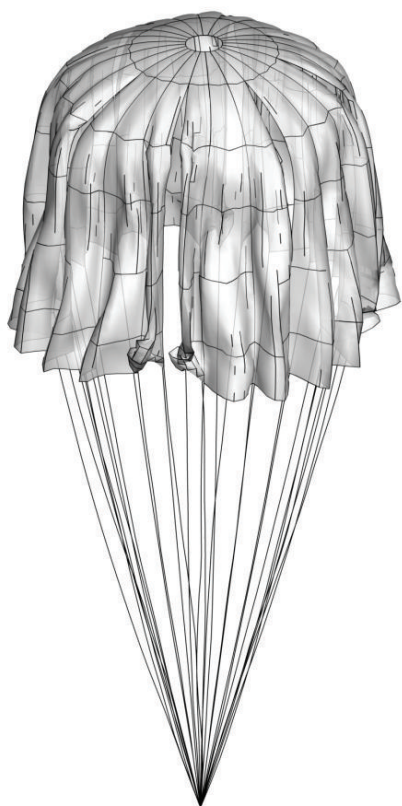

(b)

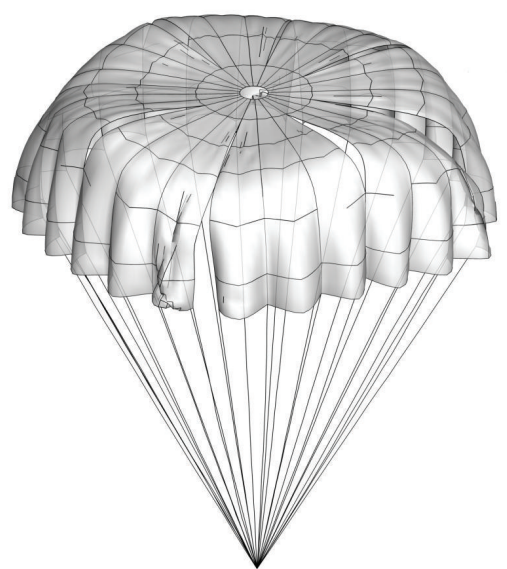

(e)

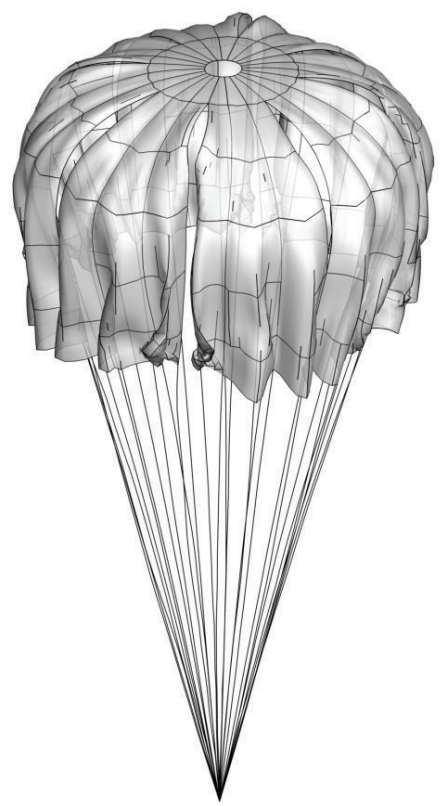

(c)

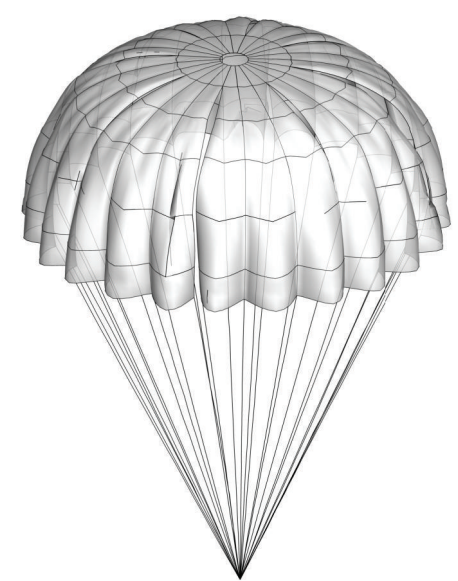

(f)

FIGURE 4: 3D canopy shape during inflation versus time: (a) 0.18, (b) 0.54, (c) 0.62, (d) 0.95, (e) 1.6, and (f) 2.0.

TABle 2: Physical parameters of dummy [14].

\begin{tabular}{lc}
\hline Name & Value \\
\hline Mass $/ \mathrm{kg}$ & 150 \\
Height/m & 2 \\
Relative height of CG/\% & 54.34 \\
$I x /\left(\mathrm{kgm}^{2}\right)$ & 0.65 \\
$I y /\left(\mathrm{kgm}^{2}\right)$ & 8.27 \\
$I z /\left(\mathrm{kgm}^{2}\right)$ & 8.71 \\
\hline
\end{tabular}

fluid was controlled according to the following dynamic refinement criteria: the regions around the folded canopy and geometrically porous area (including apex and slots) were refined to the finest resolution; in addition, the regions covered by the turbulent wakes, behind the dummy body and canopy, were refined to the finest resolution. Thus, the mesh resolution was adequate to model the structure.

\section{Results and Discussion}

The coupled numerical model simulates the FSI performance of finite mass inflation and trajectory motion of a typical slot-parachute that is used for personnel/cargo or in airdrop experiments. The obtained numerical results of inflation with $50 \mathrm{~ms}^{-1}$ initial velocity at $800 \mathrm{~m}$ height were compared to the experimental results and two other cases with different initial velocity.

3.1. Opening Process. Parachutes are stochastic systems with large scattering of their performance characteristics, and various parameters can affect their inflating performance dramatically. However, we did not consider the influence of stochastic wind in this study. Figures 4(a)-4(f) show the 3D deformation of the canopy from its initial stretched shape at $0.0 \mathrm{~s}$ up to $2.0 \mathrm{~s}$, when the canopy was completely inflated. 


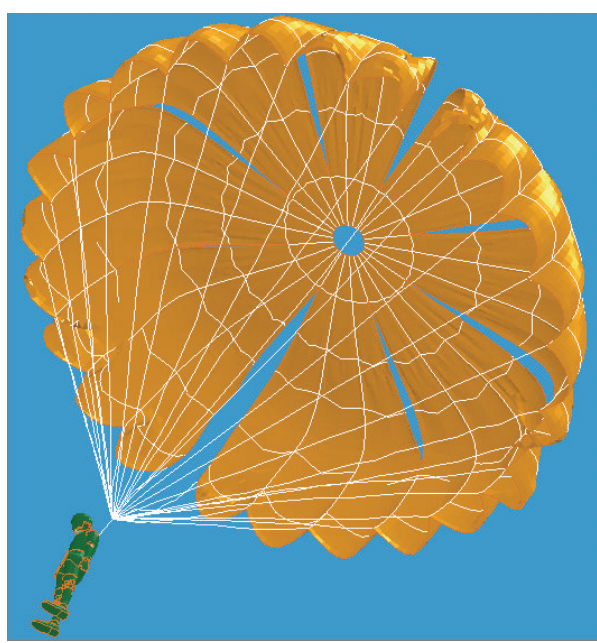

(a)

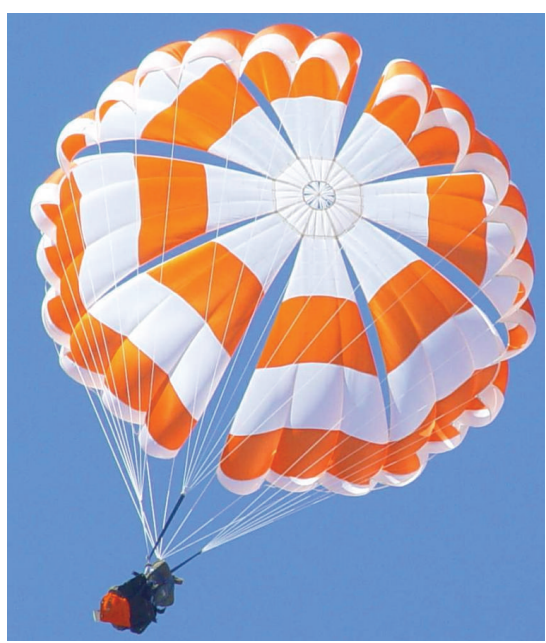

(b)

FIGURE 5: Comparison of parachute-load systems' steady state ((a) is simulation results and (b) is experimental results).

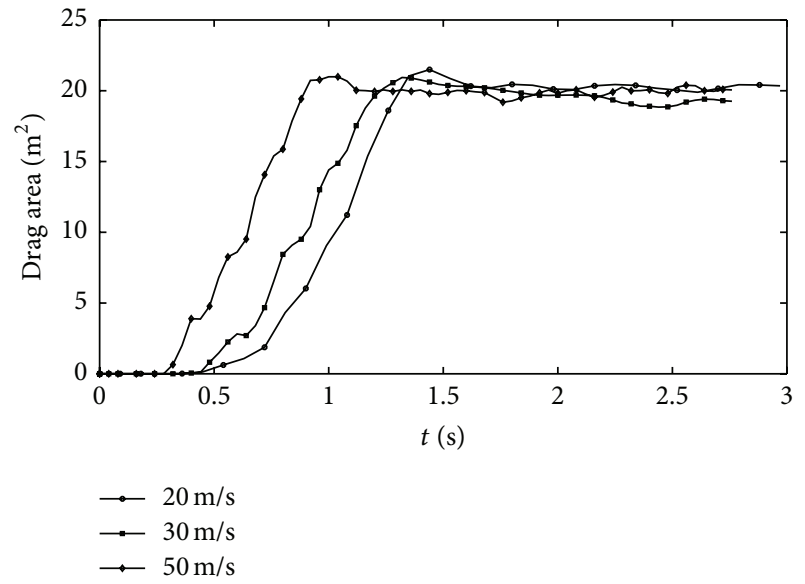

FIGURE 6: Canopy projected area versus time.

At the initial stage of inflation, the slots are narrow and long; when the airstreams gathered at the top of canopy and began to diffuse from the apex to skirt, the dimension of slots increased as the canopy expanded. Figure 9(b) shows a concavity appearing in the top of canopy for a short period, after which the flow turns the parachute into a hemisphere, indicating that the parachute comes to a steady descent state. Because of the slot-design of the fabric, the breathe phenomenon is not apparent in this type of parachute; however, the skirt slightly shrinks because of the inertial effect of the unsteady flow. Finally, we computed a convergence in the deformation results of the canopy structure to a steadily inflating state, as shown in Figure 5. The left of the figure displays the simulation results, and the right shows the photograph of parachute-dummy airdrop test.

The aforementioned results show that the numerical simulation has satisfactorily captured the opening process of the parachute; however, to better understand the decelerating behavior of parachute-load systems, some key factors should be measured and analyzed. Figure 6 shows the drag areas of three other parachute projections; the canopy reached the

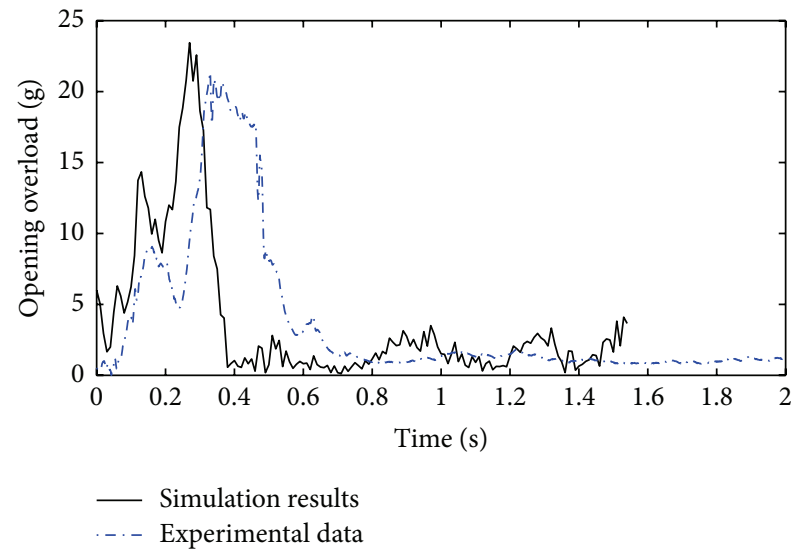

FIGURE 7: Drag force versus time (numerical and experimental).

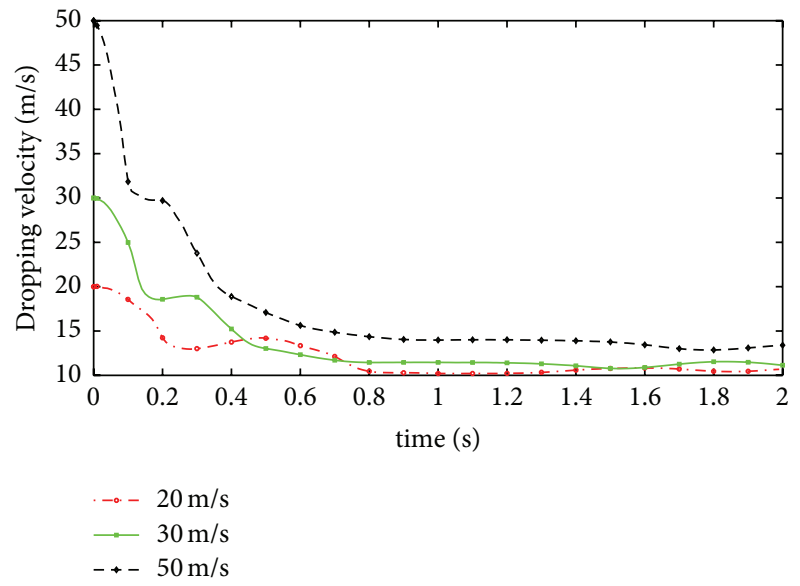

FIGURE 8: Parachute-load system's velocity comparison versus time.

maximum area and then slightly slipped and tended to keep steady. The higher initial velocity during free fall implies a shorter time for canopy opening.

Figure 7 plots the numerically predicted load acceleration versus time curve and experimental curves from the airdrop 

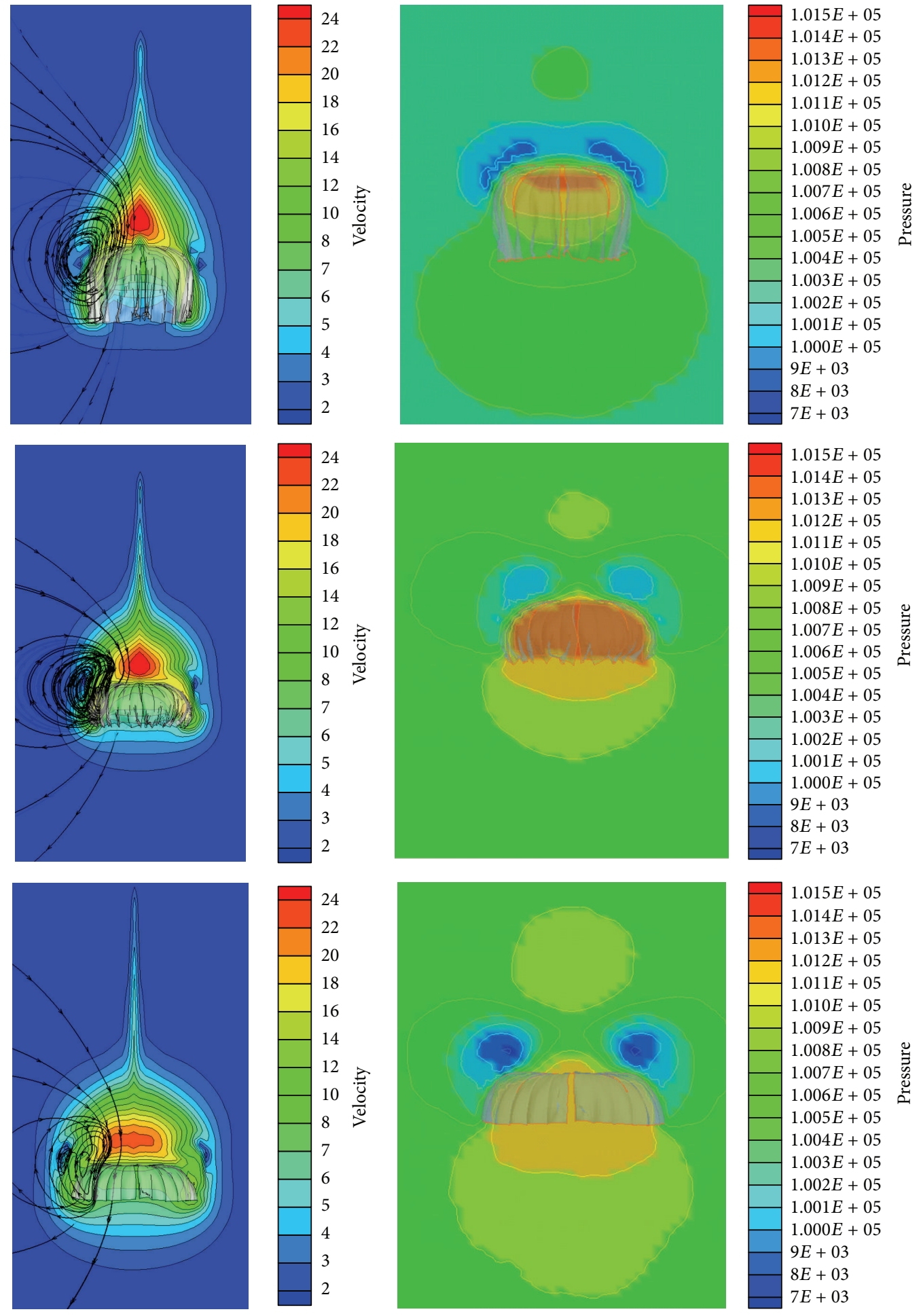

(a)

(b)

FIGURE 9: CFD pressure distributions and velocity vectors contours during opening ((a) is velocity vectors results and (b) is pressure distribution results). 


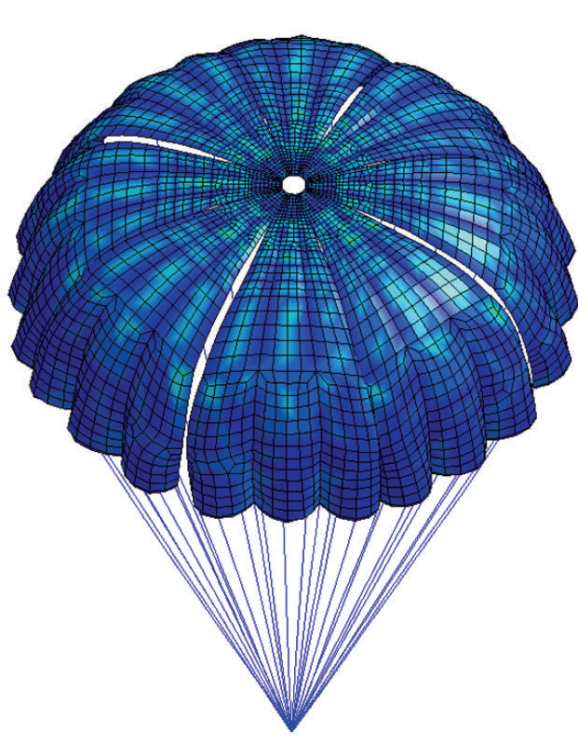

(a)

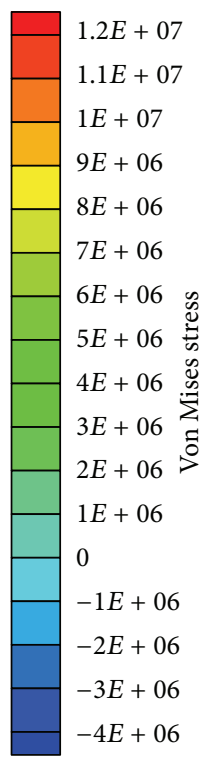

$4 E+06$

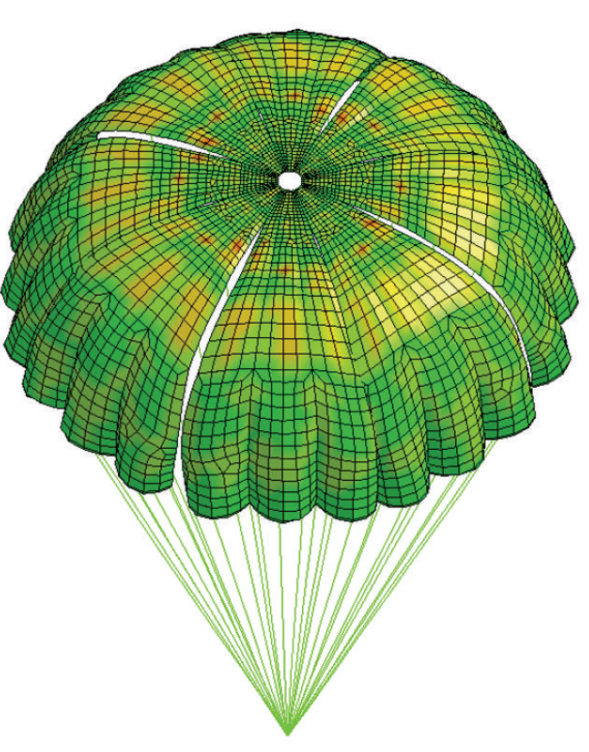

(b)

Figure 10: Von Mises stress and equivalent plastic strain distributions during opening ((a) is Von Mises stress results and (b) is equivalent plastic strain results).

test. The weight of load was $300 \mathrm{~kg}$, and the peak overload of the simulation and experiment were 23.44 and $21.36 \mathrm{~g}$, respectively. The figure shows that these two curves are very close, considering the approximations used in the model. The numerical and experimental results differ by approximately $0.1 \mathrm{~s}$ in their initiation; that is, the numerical curve starts $0.1 \mathrm{~s}$ ahead of the experimental curve. This is because of the representation of the initial shape of canopy in the FSI model that has a larger volume than the experimental canopies. Moreover, we did not entirely consider the damping effect of the canopy, which has a strong effect on the peak force value in the numerical models in this study. The larger the damping constant is, the lower the numerically predicted peak force is.

The instant velocity when canopy is stretched is equal to the initial velocity of opening. Figure 8 shows the velocity change of the parachute-load system. The velocities reduced rapidly at the beginning of the opening process for high overloads and then tended to keep steady with the balance between the drag and aerodynamic forces on the canopy. These steady velocities could be used as the initial velocities of the nine degrees of freedom models to compute the trajectory of the parachute-load system.

The FSI model also predicts a phenomenon known as wake recontact, shown in Figure 9. Wake recontact can occur in finite mass openings during or soon after the load has undergone maximum deceleration. The wake trailing the opening canopy starts moving close to the speed of the load. As a result, when the load undergoes its maximum deceleration, the wake contacts the apex of the canopy. The recontacting wake results in a negative differential pressure that indents the apex of the canopy. This phenomenon can also be seen in Figure 4(e).

The structure response of the canopy under aerodynamic pressure can also be seen in Figure 10. From the distribution of Von Mises stress and the equivalent plastic strain on the canopy, we observed that the red area near the top of canopy experiences high levels of fabric strength. The central area of each gore also suffers higher tensile strength than the average level of the whole canopy.

\section{Conclusions}

This paper presents the FSI phenomenon of a parachute during finite mass inflation with low speed and altitude. The results of the numerical model were compared with the experimental results obtained from the airdrop test, and the curves from different conditions of numerical models were compared and investigated. The ALE technique is capable of reproducing the FSI phenomenon of a parachute during opening process. Both the SD of canopy and fluid field evolvement around parachute were visually simulated and analyzed. The changing rules of the shape and overload of the canopy were in good agreement with experimental data, which prove the good inflating performance of this type of slot-parachute.

As mentioned earlier, this numerical approach can be a part of the integrated simulation system of parachute airdrop and is now being increasingly studied. Future studies should consider much more uncertainties and probabilities in environmental factors, such as gust.

\section{Competing Interests}

The authors declare that they have no competing interests.

\section{Acknowledgments}

This study was cosupported by Research Project of Chinese National University of Defense Technology (no. JC13-01-04) 
and the National Natural Science Foundation of China (Grant nos. 51375486 and 11272345).

\section{References}

[1] K. E. French, "The initial phase of parachute inflation," in Proceedings of the AIAA 2nd Aerodynamic Deceleration Systems Conference, El Centro, Calif, USA, September 1968.

[2] J. M. Macha, "A simple, approximate model of parachute inflation," AIAA Journal, pp. 44-53, 1993.

[3] D. Wolf, "Simplified dynamics model of parachute inflation," in Proceedings of the AIAA 4th Aerodynamics Deceleration Systems Conference, Palm Springs, Calif, USA, 1973.

[4] H. Johari and K. Desabrais, "A coupled fluid-structure parachute inflation model," in Proceedings of the 7th AIAA Aerodynamic Decelerator Systems Technology Conference and Seminar, Monterey, Calif, USA, May 2003.

[5] R. J. Benney and K. R. Stein, "Computational fluid-structure interaction model for parachute inflation," Journal of Aircraft, vol. 33, no. 4, pp. 730-736, 1996.

[6] K. Stein, R. Benney, T. E. Tezduyar, and J. Potvin, "Fluidstructure interactions of a cross parachute: numerical simulation," Computer Methods in Applied Mechanics and Engineering, vol. 191, no. 6-7, pp. 673-687, 2001.

[7] B. Tutt, S. Roland, G. Noetscher, and R. Charles, "Finite mass simulation techniques in LS-DYNA," in Proceedings of the 21th AIAA Aerodynamic Decelerator Systems Technology Conference and Seminar, Dublin, Ireland, May 2011.

[8] J.-D. Kim, Y. Li, and X. L. Li, "Simulation of parachute FSI using the front tracking method," Journal of Fluids and Structures, vol. 37, pp. 100-119, 2013.

[9] Y. X. Fan and J. Xia, "Simulation of 3D parachute fluidstructure interaction based on nonlinear finite element method and preconditioning finite volume method," Chinese Journal of Aeronautics, vol. 27, no. 6, pp. 1373-1383, 2014.

[10] L. Yu, H. Cheng, Y. N. Zhan, and S. Li, "Study of parachute inflation process using fluid-structure interaction method," Chinese Journal of Aeronautics, vol. 27, no. 2, pp. 272-279, 2014.

[11] G. Strickert, "Study on the relative motion of parafoil-loadsystems," Aerospace Science and Technology, vol. 8, no. 6, pp. 479-488, 2004.

[12] A. P. Taylor, "The DCLDYN parachute inflation and trajectory analysis tool-an overview," in Proceedings of the 18th AIAA Aerodynamic Decelerator Systems Technology Conference and Seminar, Munchen, Germany, 2005.

[13] K. Stein, R. Benney, V. Kalro, T. E. Tezduyar, J. Leonard, and M. Accorsi, "Parachute fluid-structure interactions: 3-D computation," Computer Methods in Applied Mechanics and Engineering, vol. 190, no. 3-4, pp. 373-386, 2000.

[14] Y. Fan, "To calculate the inertia parameter of the moving human body by means of shift matrix," Journal of Ergonomics, vol. 13, no. 1, pp. 21-24, 2007.

[15] X. L. Gao, Q. B. Zhang, and Q. G. Tang, “Transient dynamic modeling and analysis of complex parachute inflation with fixed payload," Journal of Aerospace Engineering, vol. 28, no. 4, Article ID 4014097, 2015.

[16] S. Ergun, "Fluid flow through packed beds," Chemical Engineering Progress, vol. 48, no. 2, pp. 89-94, 1952. 


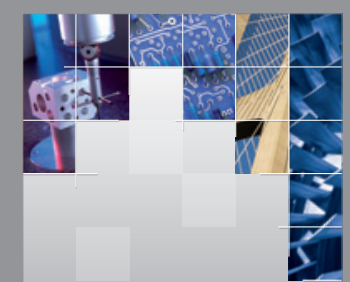

\section{Enfincering}
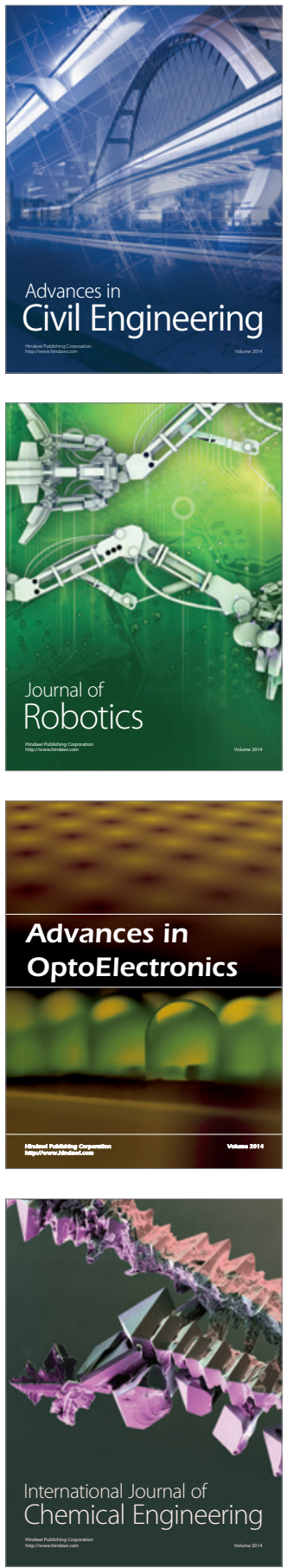

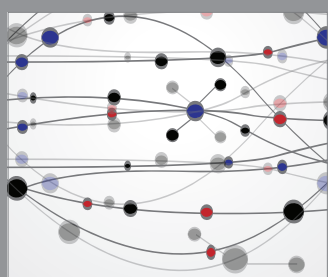

The Scientific World Journal

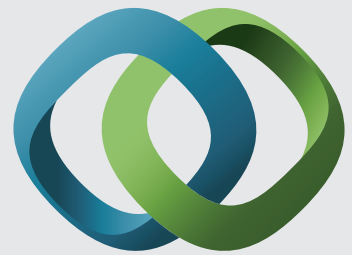

\section{Hindawi}

Submit your manuscripts at

http://www.hindawi.com
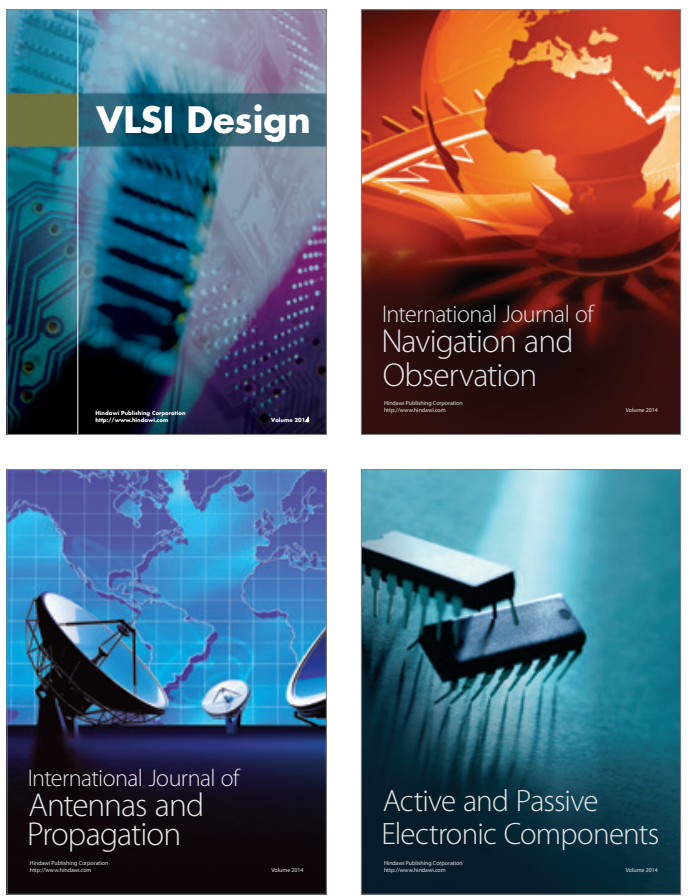
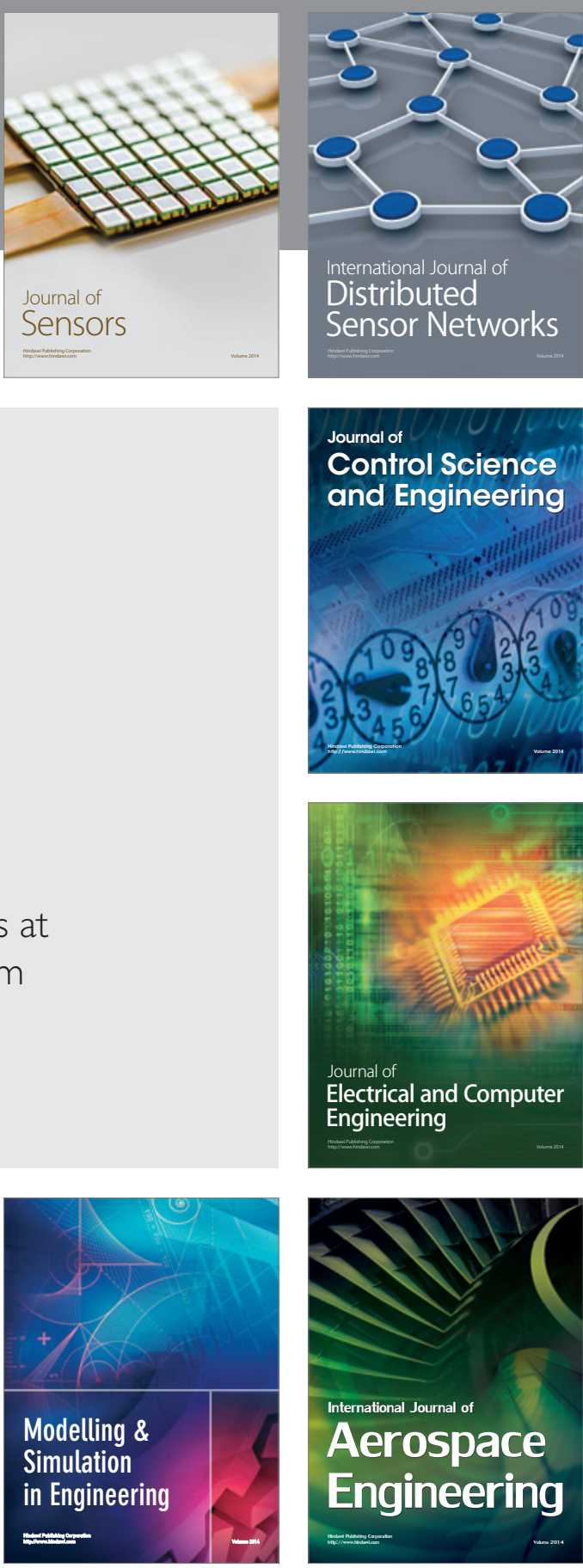

International Journal of

Distributed

Sensor Networks

Journal of

Control Science

and Engineering
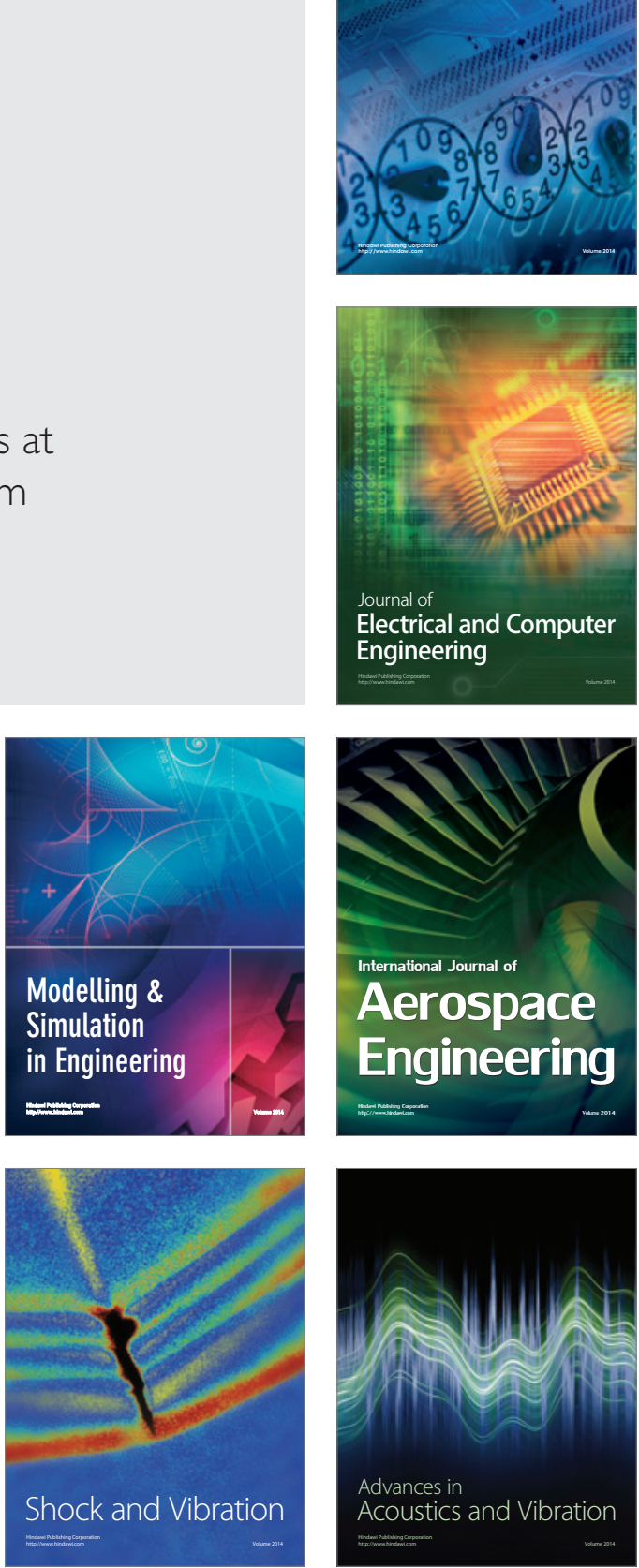